\title{
TERA-MIR radiation: materials, generation, detection and applications
}

\author{
M. F. Pereira
}

Received: 22 January 2014 / Accepted: 23 January 2014 / Published online: 8 February 2014

C) Springer Science+Business Media New York 2014

\begin{abstract}
This special edition is dedicated to original papers covering topics with the areas of interest of COST ACTION MP1204 whose main objective is to advance novel materials, concepts and device designs for generating and detecting $\mathrm{THz}(0.3-10 \mathrm{THz})$ and $\mathrm{Mid}$ Infrared $(10-100 \mathrm{THz})$ radiation using semiconductor, superconductor, metamaterials and lasers and to beneficially exploit their common aspects within a synergetic approach. The results achieved benefit from the unique networking and capacity-building capabilities provided by the COST framework to unify these two spectral domains from their common aspects of sources, detectors, materials and applications. We are building a platform to investigate interdisciplinary topics in Physics, Electrical Engineering and Technology, Applied Chemistry, Materials Sciences and Biology and Radio Astronomy. In this sense THz and MIR are considered jointly, the driving force for both regimes being applications. The main emphasis of the research presented here is on new fundamental material properties, concepts and device designs that are likely to open the way to new products or to the exploitation of new technologies in the fields of sensing, healthcare, biology, and industrial applications. End users are: research centres, academic, well-established and start-up companies and hospitals. The strong coupling of $\mathrm{THz}$ radiation and material excitations has potential to improve the quantum efficiency of $\mathrm{THz}$ devices.
\end{abstract}

Keywords TERA-MIR · Midinfrared - Terahertz - Sources · Detectors · Materials · Polaritons · Antipolaritons

In the last few years, MIR lasers, light-emitting diodes, detectors and frequency converters have featured significantly improved wavelength tunability, high spectral purity, higher powers and room temperature operation, which are necessary for commercial applications (Tittel et al. 2011; Razeghi 2013). However, whereas miniaturised and cost-efficient optical technology for wavelengths up to about $2.5 \mu \mathrm{m}$ has become readily available at a very high standard, sources for longer wavelength applications have not yet reached the desired

M. F. Pereira $(\bowtie)$

Material and Engineering Research Institute, Sheffield Hallam University, Sheffield, UK

e-mail: M.Pereira@shu.ac.uk 
standards. From the MIR detection point of view, there is a need for phase sensitive, and near single-photon sensitivity similar to that found in the near-IR (optical metrology and telecommunications) and conventional bolometric detection is one of the technologies to be ultimately replaced. At the lower end of $\mathrm{THz}$ frequencies, the microelectronic technologies with key components such as Gunn diodes, multipliers, Schottky detectors are quite successful, but at the high-frequency side, the THz-generation and detection efficiencies decrease because of RC and possibly transit-time limitations in the devices. Therefore, such devices will probably not be able to get deep into the THz frequency range. Additionally, the devices have a rather narrow tuning bandwidth, which makes them not very practical in many situations. There is much hope that intersubband devices like quantum cascade lasers (QCLs) will be the main compact $\mathrm{THz}$ source but they currently require cryogenic cooling. We will stimulate the development of more efficient simulation tools (Schmielau and Pereira 2009a,b; David 2012) and Round Robin actions for a systematic comparison of theory and experiments (Pereira et al. 2007). Thus out of the box solutions for THz QCLs such as lasing without inversion (Pereira 2008; Pereira and Tomić 2011) as well as alternative generation methods will be investigated. From the detection point of view, more advanced solutions of e.g. QuantumWell detectors for the THz range (QWIPS) require further investigation and optimization for lower costs and efficiency in a broad range. Recent Field Effect Transistor based $\mathrm{THz}$ detectors open new possibilities of construction of real time $\mathrm{THz}$ imaging systems. Their efficiency in comparison with (QWIPS) or Schottky detectors needs still be confirmed. Another possibility for room temperature $\mathrm{THz}$ sources or detectors are nitride based high electron mobility transistors (HEMT). Nitride-based devices are very prospective for device applications, since the structures are robust and can work in harsh environments and elevated temperatures. New concepts based on bismides for MIR and THz will also be investigated as well MIR applications of Chalcogenide glasses, which are based on one or more of the Chalcogen Group elements other than Oxygen, such as S, Se, and Te. They are amorphous semiconductors and have high linear and non-linear refractive indices, with large third order non-linear susceptibilities, low phonon energies. Their wide transmission window, which extends from the mid-visible to MIR, makes them very promising candidates for integrated devices for all-optical processing and biosensing. GaAsBi structures exhibit subpicosecond carrier lifetimes and have been already used effectively as photoconductors for generating $\mathrm{THz}$ radiation using pulsed lasers. However, GaSb-bismide-based materials and structures are far less known and will be grown and investigated for MIR applications (Song et al. 2013). The limits of QCL science and technology (Miriam 2012) will be investigated combining academic and industrial teams. Graphene is also emerging as a spectacular $\mathrm{THz}$ material and we plan to further stimulate joint efforts within the action (Vicarelli et al. 2012). Finally, integrating MIR components on a single chip to create a sensor system-on-a-chip and evaluating it is a task are also spectacular goals that we hope to see come through in the near future and that will be stimulated by our network.

This special edition summarizes some of the recent results of our network from fundamental Physics to applications. A wide range of related topics are covered such as: Numerical investigations of mid-infrared emission from Pr3+ doped GeAsGaSe fibre, studies of Fused fibre components for the eye-safe spectral region around $2 \mu \mathrm{m}$; Validation of protein structural models using THz spectroscopy, which is a promising approach to solve three-dimensional structures; Detailed non-destructive evaluation of UHMWPE composites in the THz range; Development of a rapid-scan fibre-integrated terahertz spectrometers; Temperature dependent nonlinear response of quantum cascade structures; Preliminary Studies of the Raman Spectra of Ag2Te and Ag5Te3; Fabrication and characterization of microlenses made of telluride and heavy metal oxide glass developed with hot embossing technology; Spontaneous 
generation of entangled exciton in quantum dot systems; Performance limits of the mid-wave InAsSb/AlAsSb nBn hot infrared detectors; Characterization of a large core photonic crystal fibre made of lead-bismuth-gallium oxide glass for broadband infrared transmission; Midinfrared supercontinuum generation in soft-glass suspended core photonic crystal fibre and the Coupling of $\mathrm{THz}$ radiation with intervalence band transitions in microcavities.

This special edition marks the kick-off our Networks' scientific activities. I thank my co-Guest Editors Anna Wójcik-Jedlinska, Trevor Benson and Marian Marciniak for their assistance in the preparation of this Special issue and we hope that our COST ACTION MP1204 initiative will help our research dreams become true.

\section{References}

Pereira Jr., M.F.: Intervalence transverse-electric mode terahertz lasing without population inversion. Phys. Rev. B. 78, 24, pp. 245305-1 -245305-5 (2008)

Pereira Jr, M.F., Nelander, R., Wacker, A., Revin, D.G., Soulby, M.R., Wilson, L.R., Cockburn, J.W., Krysa, A.B., Roberts, J.S., Airey, R.J.: Characterization of intersubband devices combining a nonequilibrium many body theory with transmission spectroscopy experiments. J. Mater. Sci.: Mater. Electron. 18, 689 (2007)

Pereira, M.F., Tomić, S.: Intersubband gain without global inversion through dilute nitride band engineering. Appl. Phys. Lett. 98, 061101 (2011)

Razeghi, M.: Quantum cascade lasers for IR and THz spectroscopy, 16 December 2013, SPIE Newsroom. doi:10.1117/2.1201312.005239

Schmielau, T., Pereira, M.F.: Impact of momentum dependent matrix elements on scattering effects in quantum cascade lasers. Phys. Status Solidi b 246, 329 (2009a)

Schmielau, T., Pereira Jr, M.F.: Nonequilibrium many body theory for quantum transport in terahertz quantum cascade lasers. Appl. Phys. Lett. 95, 231111 (2009b)

Song, Y., Gu, Y., Shao, J., Wang, S.: Dilute Bismides for Mid-IR Applications. Bismuth-Containing Compounds, pp. 1-27. ISBN/ISSN: 978-1-4614-8121-8 (2013)

Tittel, F.K., Curl, R.F., Dong, L., Doty, J.H., Kosterev, A.A., Lewicki, R., Thomazy, D., Wysocki, G.: Recent advances in infrared semiconductor laser based chemical sensing technologies. In: Pereira, M.F., Shulika, O. (eds.) Terahertz and Mid Infrared Radiation: Generation, Detection and Applications. NATO Science for Peace and Security Series B: Physics and Biophysics, 2011,doi:10.1007/978-94-007-0769-624

Vicarelli, L., Vitiello, M.S., Coquillat, D., Lombardo, A., Ferrari, A.C., Knap, W., Polini, M., Pellegrini, V., Tredicucci, A.: Graphene field-effect transistors as room-temperature terahertz detectors. Nat. Mater. 11, 865-871 (2012)

Vitiello, M.S., Consolino, L., Bartalini, S., Taschin, A., Tredicucci, A., Inguscio, M., De Natale, P.: Quantumlimited frequency fluctuations in a terahertz laser. Nat. Photon. 6, 525 (2012)

Winge, D.O., Lindskog, M., Wacker, A.: Nonlinear response of quantum cascade structures. Appl. Phys. Lett. 101, $211113(2012)$ 